07,12

\title{
Аналитическое выражение для распределения упругой деформации, создаваемой включением в форме многогранника с произвольной собственной деформацией
}

\author{
(C) A.B. Ненашев ${ }^{1}$, А.В. Двуреченский ${ }^{2}$ \\ ${ }^{1}$ Институт фризики полупроводников им. А.В. Ржанова СО РАН, \\ Новосибирск, Россия \\ ${ }^{2}$ Новосибирский государственный университет, \\ Новосибирск, Россия \\ E-mail: nenashev@isp.nsc.ru
}

(Поступила в Редакцию 28 февраля 2018 г.)

Получены аналитические выражения для вектора смещения, тензора деформации и тензора Эшелби в случае, когда включение в упруго-изотропной бесконечной среде имеет форму многогранника. Собственная деформация (например, рассогласование кристалических решеток) предполагается постоянной внутри включения, но не обязательно гидростатической. Найденные выражения описывают деформацию как внутри включения, так и в его окружении. Показано, что сложная трехмерная конфигурация поля упругой деформации (а также вектора смещения) сводится к комбинации простых функций, имеющих наглядную физическую и геометрическую интерпретацию.

Работа профинансирована Российским научным фондом (грант 14-12-00931 П).

DOI: 10.21883/FTT.2018.09.46394.053

\section{1. Введение}

Круг задач, связанных с распределением деформации, индуцированной включением в упругой среде, изучается в течение более чем полувека $[1,2]$, однако и в настоящее время появляются новые результаты (обзор современного состояния можно найти в работах [3-6]). Одним из важных применений задачи о включении является исследование электронных свойств полупроводниковых квантовых точек, образующихся в процессе эпитаксиального роста [7-10]. Деформации изменяют положение краев энергетических зон в полупроводниках $[11,12]$ и тем самым модифицируют энергетический спектр носителей заряда. Особенно ярко этот эффект проявляется в гетеросистемах 2-го типа, таких как германий-кремний, где деформация приводит к возникновению потенциальных ям для электронов в окрестности Ge квантовых точек [10-13].

Источником деформации в полупроводниковых гетеросистемах является разница между постоянными решетки включения $(I)$ и матрицы $(M)$, как схематично показано на рис. 1, $a$. Это различие можно интерпретировать как наличие собственной дефбормащии $\varepsilon_{i j}^{(0)}$ в материале включения, равной

$$
\begin{gathered}
\varepsilon_{x x}^{(0)}=\varepsilon_{y y}^{(0)}=\varepsilon_{z z}^{(0)}=\frac{a_{I}-a_{M}}{a_{M}}, \\
\varepsilon_{x y}^{(0)}=\varepsilon_{x z}^{(0)}=\varepsilon_{y z}^{(0)}=0,
\end{gathered}
$$

где $a_{M}$ и $a_{I}$ - равновесные параметры решеток матрицы и включения. Когда включение оказывается встроенным в матрицу, происходит перераспределение деформации, которое можно описать как перемещение материала $\mathbf{u}(\mathbf{r})$ под действием сил, приложенных к границе включения, см. рис. $1, c$.
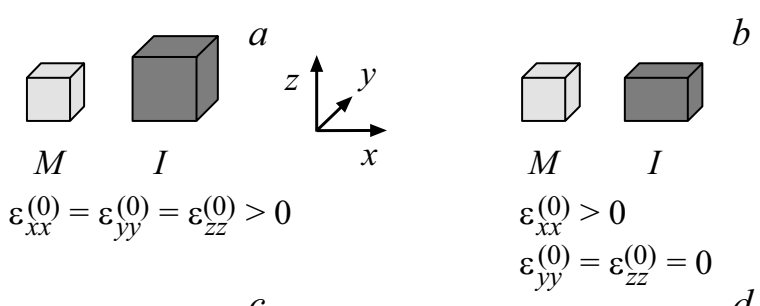

$c$
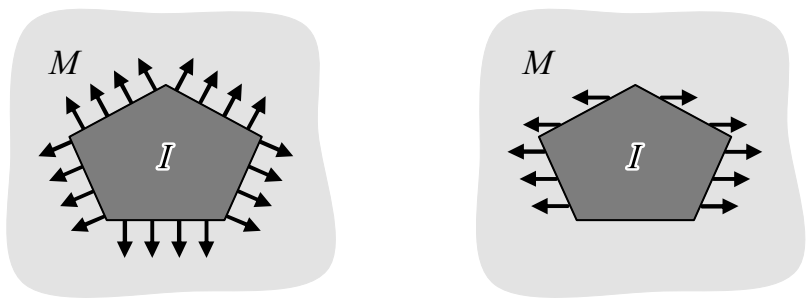

Рис. 1. Иллюстрация различия между гидростатической $(a, c)$ и негидростатической $(b, d)$ собственной деформацией. $a-$ элементарный объем матрицы $(M)$ и включения $(I)$ в релаксированном состоянии в случае гидростатической собственной деформации (материал включения растянут по всем трем направлениям); $b$ - то же для негидростатической собственной деформации, в частном случае когда материал включения растянут только по оси $X$. Для наглядности растяжение преувеличено; $c, d-$ поверхностные силы, действие которых описывает упругую релаксацию системы „матрица + включение“, в случае гидростатической $(c)$ и негидростатической $(d)$ собственной деформации. 
На рис. 1, $a, c$ показан случай гидростатической (дилатационной) собственной деформации, когда тензор $\varepsilon_{i j}^{(0)}$ изотропен (как в случае рассогласования решеток между кубическими полупроводниками). Рис. $1, b, d$ демонстрирует пример негидростатической собственной деформации. В этом иллюстративном примере различие между параметрами решетки двух сред имеет место только в направлении оси $X$. Случай негидростатической собственной деформации, помимо самостоятельного значения, возникает в задаче об упругой неоднородности (т.е. включения с модулями упругости, отличными от соответствующих модулей матрицы) как поправка по теории возмущений в рамках метода эффективного включения $[14,15]$.

Целью настоящего исследования является разработка аналитического метода для нахождения пространственного распределения деформации $\varepsilon_{i j}(\mathbf{r})$, создаваемой включением, имеющим форму произвольного многогранника, с произвольной (гидростатической или негидростатической) собственной деформацией $\varepsilon_{i j}^{(0)}$. Материалы матрицы и включения предполагаются упругоизотропными и имеющими одинаковые модули упругости. Для простоты мы рассмотрим лишь случай постоянной собственной деформации. Это, однако, не является ограничением метода: результаты нашей предыдущей работы [5] позволяют обобщить рассмотрение на случай плавно меняющейся в пространстве (полиномиальным образом) собственной деформации. В литературе имеются аналитические решения для случая постоянной собственной деформации [16,17], однако они чрезвычайно сложны и не допускают непосредственного обобщения на случай зависящей от координат собственной деформации. Между тем последний случай важен, во-первых, потому что реальные включения (такие как полупроводниковые квантовые точки) часто имеют плавно меняющийся в пространстве состав [18-20], а во-вторых, потому что задача с переменной собственной деформацией возникает при использовании упомянутого выше метода эффективного включения.

\section{2. Теория}

Математическая постановка задачи выглядит следующим образом. Тензор деформации $\varepsilon_{i j}$ как функция координат выражается в виде

$$
\varepsilon_{i j}(\mathbf{r})=\frac{1}{2}\left(\frac{\partial u_{i}(\mathbf{r})}{\partial r_{j}}+\frac{\partial u_{j}(\mathbf{r})}{\partial r_{i}}\right)-\varepsilon_{i j}^{(0)} \chi(\mathbf{r}),
$$

где $\chi(\mathbf{r})=1$ внутри включения и $\chi(\mathbf{r})=0$ снаружи. Итоговое распределение деформации определяется уравнениями равновесия, которые имеют вид

$$
\frac{\partial \sigma_{i j}(\mathbf{r})}{\partial r_{j}}=0
$$

где $\sigma_{i j}-$ тензор напряжений, выражаемый через тензор деформации $\varepsilon_{i j}$ и упругие константы (коэффициенты Ламэ) $\lambda$ и $\mu$ посредством закона Гука

$$
\sigma_{i j}(\mathbf{r})=\lambda \delta_{i j} \varepsilon_{m m}(\mathbf{r})+2 \mu \varepsilon_{i j}(\mathbf{r}) .
$$

Как показано в классической работе Эшелби [1], решение уравнений равновесия в данном случае есть комбинация производных от ньютоновского потенциала $\varphi(\mathbf{r})$, создаваемого в точке $\mathbf{r}$ притягивающим веществом, однородно заполняющим объем включения

$$
\varphi(\mathbf{r})=\iiint_{V} \frac{d V^{\prime}}{\left|\mathbf{r}-\mathbf{r}^{\prime}\right|},
$$

и производных „бигармонического потенциала“ $U(\mathbf{r})$, определяемого как

$$
U(\mathbf{r})=\iiint_{\mathrm{V}}\left|\mathbf{r}-\mathbf{r}^{\prime}\right| d V^{\prime}
$$

Согласно [1], вектор смещения $\mathbf{u}(\mathbf{r})$ равен

$$
u_{i}(\mathbf{r})=-\frac{\sigma_{j k}^{(0)}}{16 \pi \mu}\left(4 \delta_{i j} \frac{\partial \varphi(\mathbf{r})}{\partial r_{k}}-\frac{1}{1-v} \frac{\partial^{3} U(\mathbf{r})}{\partial r_{i} \partial r_{j} \partial r_{k}}\right),
$$

где $\sigma_{i j}^{(0)}$ - тензор напряжений, соответствующий собственной деформации $\varepsilon_{i j}^{(0)}$ согласно (4), а $v-$ коэффициент Пуассона. Выражая $\sigma_{i j}^{(0)}$ через $\varepsilon_{i j}^{(0)}$ и учитывая, что $\varphi=\frac{1}{2} \Delta U$ и $\lambda=2 \mu \nu /(1-2 v)$, получим

$$
\begin{aligned}
u_{i}(\mathbf{r})= & -\frac{1}{8 \pi}\left[2 \varepsilon_{i k}^{(0)} U_{, k l l}(\mathbf{r})+\frac{v}{1-v} \varepsilon_{k k}^{(0)} U_{, i l l}(\mathbf{r})\right. \\
& \left.-\frac{1}{1-v} \varepsilon_{k l}^{(0)} U_{, i k l}(\mathbf{r})\right] .
\end{aligned}
$$

(Здесь мы используем сокращенное обозначение $U_{, i j k}$ для производных $\partial^{3} U / \partial r_{i} \partial r_{j} \partial r_{k}$.) Видно, что компоненты вектора смещения $\mathbf{u}$ являются линейными комбинациями третьих производных „бигармонического потенциала“" $U$.

Подставляя (8) в (2), находим, что компоненты тензора деформации $\varepsilon_{i j}$ представляются в виде комбинаций четвертых производных от $U$

$$
\begin{aligned}
\varepsilon_{i j}(\mathbf{r})= & -\frac{1}{8 \pi}\left(\varepsilon_{i k}^{(0)} U_{, j k l l}+\varepsilon_{j k}^{(0)} U_{, i k l l}+\frac{v}{1-v} \varepsilon_{k k}^{(0)} U_{, i j l l}\right. \\
& \left.-\frac{1}{1-v} \varepsilon_{k l}^{(0)} U_{, i j k l}\right)-\varepsilon_{i j}^{(0)} \chi(\mathbf{r})
\end{aligned}
$$

Коэффициенты пропорциональности между компонентами тензоров $\varepsilon_{i j}$ и $\varepsilon_{k l}^{(0)}$ составляют так называемый тензор Эшелби $S_{i j k l}$, симметричный по первой и по второй парам индексов:

$$
\varepsilon_{i j}(\mathbf{r})=S_{i j k l}(\mathbf{r}) \varepsilon_{k l}^{(0)}-\chi(\mathbf{r}) \varepsilon_{i j}^{(0)} .
$$


Сравнивая (9) с (10), находим следующее выражение для тензора $S_{i j k l}$ через производные $U$

$$
\begin{aligned}
S_{i j k l}(\mathbf{r})= & -\frac{1}{8 \pi}\left[\frac { 1 } { 2 } \left(\delta_{i k} U_{, j l m m}+\delta_{j k} U_{, i l m m}+\delta_{i l} U_{, j k m m}\right.\right. \\
& \left.\left.+\delta_{j l} U_{, i k m m}\right)+\frac{v}{1-v} \delta_{k l} U_{, i j m m}-\frac{1}{1-v} U_{, i j k l}\right] .
\end{aligned}
$$

Итак, вычисление вектора смещения $\mathbf{u}(\mathbf{r})$, тензора деформации $\varepsilon_{i j}(\mathbf{r})$ и тензора Эшелби $S_{i j k l}(\mathbf{r})$ сводится к нахождению аналитических выражений для третьих и четвертых производных потенциала $U(\mathbf{r})$. В настоящей работе получены такие аналитические выражения (30) и (33), имеющие достаточно простой и компактный вид, для случая включения в форме многогранника.

Для этого мы применили метод, использованный нами в работах [21,5] и аналогичный методу Кувшинова [4]. А именно, мы представили требуемые выражения в виде комбинаций некоторых достаточно простых двумерных и одномерных интегралов по граням и ребрам поверхности включения. Для целей данной работы нам понадобятся следующие интегралы (индекс $\alpha$ нумерует грани, $\beta-$ ребра, а $\gamma-$ вершины поверхности)

$$
\tilde{\Phi}_{\alpha}(\mathbf{r})=\iint_{\text {on facet } \alpha}\left|\mathbf{r}-\mathbf{r}^{\prime}\right| d S^{\prime},
$$

где $d S^{\prime}$ - элемент площади грани, по которой пробегает вектор $\mathbf{r}^{\prime}$,

$$
\begin{gathered}
\Phi_{\alpha}(\mathbf{r})=\iint_{\text {on facet } \alpha} \frac{1}{\left|\mathbf{r}-\mathbf{r}^{\prime}\right|} d S^{\prime}, \\
\Omega_{\alpha}(\mathbf{r})=\iint_{\text {on facet } \alpha} \frac{\left(\mathbf{r}-\mathbf{r}^{\prime}\right) \cdot \mathbf{n}_{\alpha}}{\left|\mathbf{r}-\mathbf{r}^{\prime}\right|^{3}} d S^{\prime},
\end{gathered}
$$

где $\mathbf{n}_{\alpha}$ - единичный вектор, направленный по нормали к грани $\alpha$ изнутри включения наружу (см. рис. 2),

$$
\tilde{L}_{\beta}(\mathbf{r})=\int_{\text {on edge } \beta}\left|\mathbf{r}-\mathbf{r}^{\prime}\right| d l^{\prime},
$$

где $d l^{\prime}$ - элемент длины ребра, по которому пробегает вектор $\mathbf{r}^{\prime}$,

$$
\begin{aligned}
L_{\beta}(\mathbf{r}) & =\int_{\text {on edge } \beta} \frac{1}{\left|\mathbf{r}-\mathbf{r}^{\prime}\right|} d l^{\prime}, \\
\Lambda_{\beta}(\mathbf{r}) & =\int_{\text {on edge } \beta} \frac{1}{\left|\mathbf{r}-\mathbf{r}^{\prime}\right|^{3}} d l^{\prime} .
\end{aligned}
$$

Покажем теперь, что третьи и четвертые производные потенциала $U(\mathbf{r})$ являются комбинациями интегралов $\Omega_{\alpha}, L_{\beta}$ и $\Lambda_{\beta}$, для которых известны простые аналитические выражения. Для этого, помимо обозначения $\mathbf{n}_{\alpha}$ для вектора нормали к грани $\alpha$, введем обозначения $\mathbf{b}_{\alpha \beta}$ для единичного вектора, направленного „изнутри наружу“
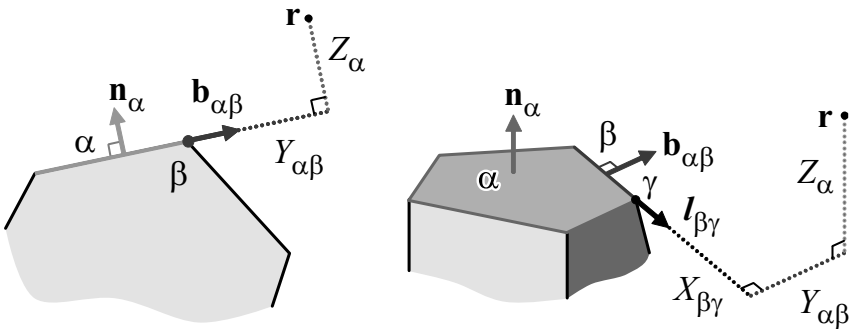

Рис. 2. Единичные векторы $\mathbf{n}_{\alpha}, \mathbf{b}_{\alpha \beta}, \mathbf{l}_{\beta \gamma}$ и компоненты радиусвектора $X_{\beta \gamma}, Y_{\alpha \beta}, Z_{\alpha}$ : проекция на плоскость, перпендикулярную ребру $\beta$ (слева) и вид сбоку (справа). $\mathbf{n}_{\alpha}-$ нормаль к грани $\alpha$; $\mathbf{b}_{\alpha \beta}$ - вектор, перпендикулярный к ребру $\beta$ и лежащий в плоскости грани $\alpha ; \mathbf{l}_{\beta \gamma}-$ вектор вдоль ребра $\beta$, направленный к вершине $\gamma$ от противоположной ей вершины.

грани $\alpha$ перпендикулярно к смежному с ней ребру $\beta$; и $\mathbf{l}_{\beta \gamma}$ для единичного вектора вдоль ребра $\beta$, выходящего из него в вершине $\gamma$ (рис. 2). Обозначим также через $Z_{\alpha}, Y_{\alpha \beta}, X_{\beta \gamma}$ проекции радиус-вектора $\mathbf{r}$, взятого относительно соответствующих элементов многогранника, на единичные векторы $\mathbf{n}_{\alpha}, \mathbf{b}_{\alpha \beta}, \mathbf{l}_{\beta \gamma}$ :

$$
\begin{gathered}
Z_{\alpha}(\mathbf{r})=\left(\mathbf{r}-\mathbf{r}_{\alpha}\right) \cdot \mathbf{n}_{\alpha}, \\
Y_{\alpha \beta}(\mathbf{r})=\left(\mathbf{r}-\mathbf{r}_{\beta}\right) \cdot \mathbf{b}_{\alpha \beta}, \\
X_{\beta \gamma}(\mathbf{r})=\left(\mathbf{r}-\mathbf{r}_{\gamma}\right) \cdot \mathbf{l}_{\beta \gamma},
\end{gathered}
$$

где $\mathbf{r}_{\alpha}, \mathbf{r}_{\beta}, \mathbf{r}_{\gamma}$ - радиус-векторы произвольно выбранных точек на грани $\alpha$ и ребре $\beta$ и радиус-вектор вершины $\gamma$ соответственно. Проекцию вектора $\mathbf{r}-\mathbf{r}_{\beta}$ на плоскость, перпендикулярную ребру $\beta$, обозначим $\mathbf{R}_{\perp \beta}$

$$
\mathbf{R}_{\perp \beta}(\mathbf{r})=Z_{\alpha} \mathbf{n}_{\alpha}+Y_{\alpha \beta} \mathbf{b}_{\alpha \beta}=\mathbf{r}-\mathbf{r}_{\beta}-\left[\left(\mathbf{r}-\mathbf{r}_{\beta}\right) \cdot \mathbf{l}_{\beta \gamma}\right] \mathbf{l}_{\beta \gamma} .
$$

С помощью этих обозначений производные по $\mathbf{r}$ от интегралов $U, \tilde{\Phi}, \Phi, \tilde{L}, L, \Omega$ выражаются в следующем виде:

$$
\frac{\partial U(\mathbf{r})}{\partial \mathbf{r}}=-\sum_{\alpha} \mathbf{n}_{\alpha} \tilde{\Phi}_{\alpha},
$$

где суммирование идет по всем граням поверхности включения;

$$
\begin{gathered}
\frac{\partial \tilde{\Phi}_{\alpha}}{\partial \mathbf{r}}=\mathbf{n}_{\alpha} Z_{\alpha} \Phi_{\alpha}-\sum_{\beta} \mathbf{b}_{\alpha \beta} \tilde{L}_{\beta}, \\
\frac{\partial \Phi_{\alpha}}{\partial \mathbf{r}}=-\mathbf{n}_{\alpha} \Omega_{\alpha}-\sum_{\beta} \mathbf{b}_{\alpha \beta} L_{\beta}, \\
\frac{\partial\left(Z_{\alpha} \Omega_{\alpha}\right)}{\partial \mathbf{r}}=\mathbf{n}_{\alpha} \Omega_{\alpha}+Z_{\alpha} \sum_{\beta}\left(\mathbf{n}_{\alpha} Y_{\alpha \beta}-\mathbf{b}_{\alpha \beta} Z_{\alpha}\right) \Lambda_{\beta},
\end{gathered}
$$

где индекс $\beta$ пробегает по ребрам, окружающим грань $\alpha$;

$$
\begin{aligned}
& \frac{\partial \tilde{L}_{\beta}}{\partial \mathbf{r}}=\mathbf{R}_{\perp \beta} L_{\beta}-\sum_{\gamma} \mathbf{l}_{\beta \gamma}\left|\mathbf{r}-\mathbf{r}_{\gamma}\right|, \\
& \frac{\partial L_{\beta}}{\partial \mathbf{r}}=-\mathbf{R}_{\perp \beta} \Lambda_{\beta}-\sum_{\gamma} \frac{\mathbf{l}_{\beta \gamma}}{\left|\mathbf{r}-\mathbf{r}_{\gamma}\right|},
\end{aligned}
$$


где индекс $\gamma$ пробегает по двум вершинам, ограничивающим ребро $\beta$;

$$
\frac{\partial\left|\mathbf{r}-\mathbf{r}_{\gamma}\right|}{\partial \mathbf{r}}=\frac{\mathbf{r}-\mathbf{r}_{\gamma}}{\left|\mathbf{r}-\mathbf{r}_{\gamma}\right|} .
$$

Кроме того, как следует из формулы (55) в работе [5], интеграл $\Phi_{\alpha}$ сводится к комбинации величин $\Omega_{\alpha}$ и $L_{\beta}$ :

$$
\Phi_{\alpha}=-Z_{\alpha} \Omega_{\alpha}-\sum_{\beta} Y_{\alpha \beta} L_{\beta},
$$

где сумма пробегает по ребрам, окружающим грань $\alpha$.

Применяя последовательно формулы (22)-(24), (26), (29), легко найти выражение для третьих производных $U_{, i j k} \equiv \partial^{3} U / \partial r_{i} \partial r_{j} \partial r_{k}$ потенциала $U(\mathbf{r})$

$$
\begin{aligned}
U_{, i j k}(\mathbf{r})= & \sum_{\alpha} 2 n_{\alpha i} n_{\alpha j} n_{\alpha k} Z_{\alpha} \Omega_{\alpha} \\
& +\sum_{\beta} p_{i j k l}^{(\beta)}\left(\mathbf{r}-\mathbf{r}_{\beta}\right)_{l} L_{\beta}+\sum_{\gamma} q_{i j k}^{(\gamma)}\left|\mathbf{r}-\mathbf{r}_{\gamma}\right|
\end{aligned}
$$

Здесь индексы $\alpha, \beta, \gamma$ пробегают по всем граням, ребрам и вершинам многогранника, соответственно. Тензоры $p_{i j k l}^{(\beta)}$ и $q_{i j k}^{(\gamma)}$ не зависят от $\mathbf{r}$ и выражаются следующим образом через единичные векторы $\mathbf{n}, \mathbf{b}, \mathbf{l}$ :

$$
\begin{aligned}
p_{i j k l}^{(\beta)}= & \sum_{\alpha}\left[n_{\alpha i} n_{\alpha j}\left(n_{\alpha k} b_{\alpha \beta l}+b_{\alpha \beta k} n_{\alpha l}\right)\right. \\
& \left.+n_{\alpha i} b_{\alpha \beta j}\left(n_{\alpha k} n_{\alpha l}+b_{\alpha \beta k} b_{\alpha \beta l}\right)\right],
\end{aligned}
$$

где суммирование происходит по двум граням, сходящимся в ребре $\beta$;

$$
q_{i j k}^{(\gamma)}=-\sum_{(\alpha, \beta)} n_{\alpha i} b_{\alpha \beta j} l_{\beta \gamma k},
$$

где суммирование имеет место по парам „грань + смежное с ней ребро“ таким, что и грань и ребро проходят через вершину $\gamma$.

Аналогично, используя (22)-(28), найдем четвертые производные потенциала $U$

$$
\begin{aligned}
& U_{, i j k l}(\mathbf{r})=\sum_{\alpha} 2 n_{\alpha i} n_{\alpha j} n_{\alpha k} n_{\alpha l} \Omega_{\alpha}+\sum_{\beta} p_{i j k l}^{(\beta)} L_{\beta} \\
& +\sum_{\beta} v_{i j k l m n}^{(\beta)}\left(\mathbf{r}-\mathbf{r}_{\beta}\right)_{m}\left(\mathbf{r}-\mathbf{r}_{\beta}\right)_{n} \Lambda_{\beta}+\sum_{\gamma} w_{i j k l m}^{(\gamma)} \frac{\left(\mathbf{r}-\mathbf{r}_{\gamma}\right)_{m}}{\left|\mathbf{r}-\mathbf{r}_{\gamma}\right|} .
\end{aligned}
$$

Входящие в эту формулу тензоры $p_{i j k l}^{(\beta)}, v_{i j k l m n}^{(\beta)}$ и $w_{i j k l m}^{(\gamma)}$ не зависят от $\mathbf{r}$ и являются комбинациями единичных векторов $\mathbf{n}, \mathbf{b}, \mathbf{l}$

$$
\begin{aligned}
v_{i j k l m n}^{(\beta)}= & \sum_{\alpha} n_{\alpha i}\left\{n_{\alpha j} n_{\alpha k} n_{\alpha m}\left(n_{\alpha l} b_{\alpha \beta n}-b_{\alpha \beta l} n_{\alpha n}\right)\right. \\
& -\left[n_{\alpha j} b_{\alpha \beta k} n_{\alpha m}+b_{\alpha \beta j}\left(n_{\alpha k} n_{\alpha m}+b_{\alpha \beta k} b_{\alpha \beta m}\right)\right] \\
& \left.\times\left(n_{\alpha l} n_{\alpha n}+b_{\alpha \beta l} b_{\alpha \beta n}\right)\right\}, \\
w_{i j k l m}^{(\gamma)}= & -\sum_{(\alpha, \beta)} n_{\alpha i}\left[n_{\alpha j} b_{\alpha \beta k} l_{\beta \gamma l} n_{\alpha m}+b_{\alpha \beta j} l_{\beta \gamma k} \delta_{l m}\right. \\
& \left.+b_{\alpha \beta j} l_{\beta \gamma l}\left(n_{\alpha k} n_{\alpha m}+b_{\alpha \beta k} b_{\alpha \beta m}\right)\right] .
\end{aligned}
$$

Суммирование в (34) и (35) происходит по тому же принципу, что и в (31) и (32) соответственно. Тензор $p_{i j k l}^{(\beta)}$ был определен выше.

Последнее, что необходимо сделать, - это выразить величины $\Omega_{\alpha}(\mathbf{r}), L_{\beta}(\mathbf{r})$ и $\Lambda_{\beta}(\mathbf{r})$, фигурирующие в (30) и (33), через элементарные функции. Этим величинам можно придать следующий наглядный смысл. $L_{\beta}(\mathbf{r})-$ это кулоновский потенциал, создаваемый равномерно заряженным (с единичной плотностью) ребром $\beta$ в точке $\mathbf{r} . \Lambda_{\beta}(\mathbf{r})$ отличается от $L_{\beta}(\mathbf{r})$ тем, что „потенциал взаимодействия“ убывает с расстоянием как $r^{-3}$, а не как $r^{-1}$. Одномерные интегралы $L_{\beta}$ и $\Lambda_{\beta}$ вычисляются элементарно

$$
\begin{aligned}
& L_{\beta}(\mathbf{r})=\ln \frac{\left|\mathbf{r}-\mathbf{r}_{\gamma_{1}}\right|+\left|\mathbf{r}-\mathbf{r}_{\gamma_{2}}\right|+\left|\mathbf{r}_{\gamma_{1}}-\mathbf{r}_{\gamma_{2}}\right|}{\left|\mathbf{r}-\mathbf{r}_{\gamma_{1}}\right|+\left|\mathbf{r}-\mathbf{r}_{\gamma_{2}}\right|-\left|\mathbf{r}_{\gamma_{1}}-\mathbf{r}_{\gamma_{2}}\right|}, \\
& \Lambda_{\beta}(\mathbf{r})=-\frac{1}{\left(\mathbf{R}_{\perp \beta}\right)^{2}}\left(\frac{X_{\beta \gamma_{1}}}{\left|\mathbf{r}-\mathbf{r}_{\gamma_{1}}\right|}+\frac{X_{\beta \gamma_{2}}}{\left|\mathbf{r}-\mathbf{r}_{\gamma_{2}}\right|}\right),
\end{aligned}
$$

где индексы $\gamma_{1}$ и $\gamma_{2}$ относятся к двум вершинам, ограничивающим ребро $\beta ; \mathbf{r}_{\gamma_{1}}$ и $\mathbf{r}_{\gamma_{2}}-$ радиус-векторы этих вершин. Наконец, $\Omega_{\alpha}(\mathbf{r})$ - это потенциал в точке $\mathbf{r}$, создаваемый дипольным слоем, равномерно распределенным по грани $\alpha$ (дипольный момент направлен в сторону нормали $\mathbf{n}_{\alpha}$ ). Как известно [22], модуль этой величины $\left|\Omega_{\alpha}(\mathbf{r})\right|$ равен телесному углу, под которым видна грань $\alpha$ из точки r. Знак $\Omega_{\alpha}(\mathbf{r})$ равен знаку $Z_{\alpha}$, т.е. положителен, если точка $\mathbf{r}$ находится с той стороны от грани $\alpha$, в которую направлена нормаль $\mathbf{n}_{\alpha}$, и отрицателен в противном случае. Явные выражения для $\Omega_{\alpha}(\mathbf{r})$ через элементарные (обратные тригонометрические) функции можно найти в [23-25] для треугольной грани и в [25] для прямоугольной грани. Грань более сложной формы можно разбить на треугольники, либо воспользоваться методом вычисления из работы [24].

\section{3. Пример}

На рис. 3 показан пример вычисления вектора смещения $\mathbf{u}(\mathbf{r})$, тензора деформации $\varepsilon_{i j}(\mathbf{r})$ и тензора напряжений $\sigma_{i j}(\mathbf{r})$ с помощью полученных нами аналитических выражений.

В качестве модельной структуры взято включение пирамидальной формы с квадратным основанием 


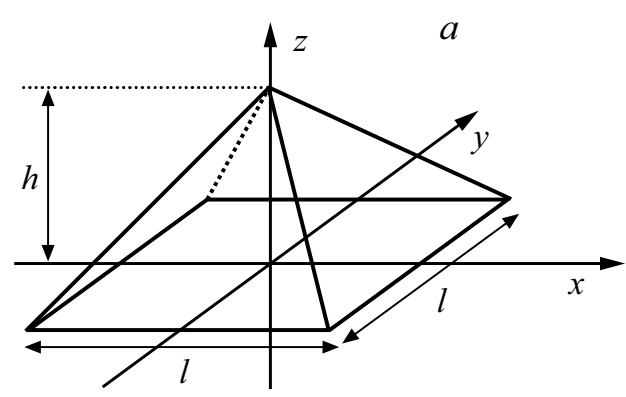

$b$
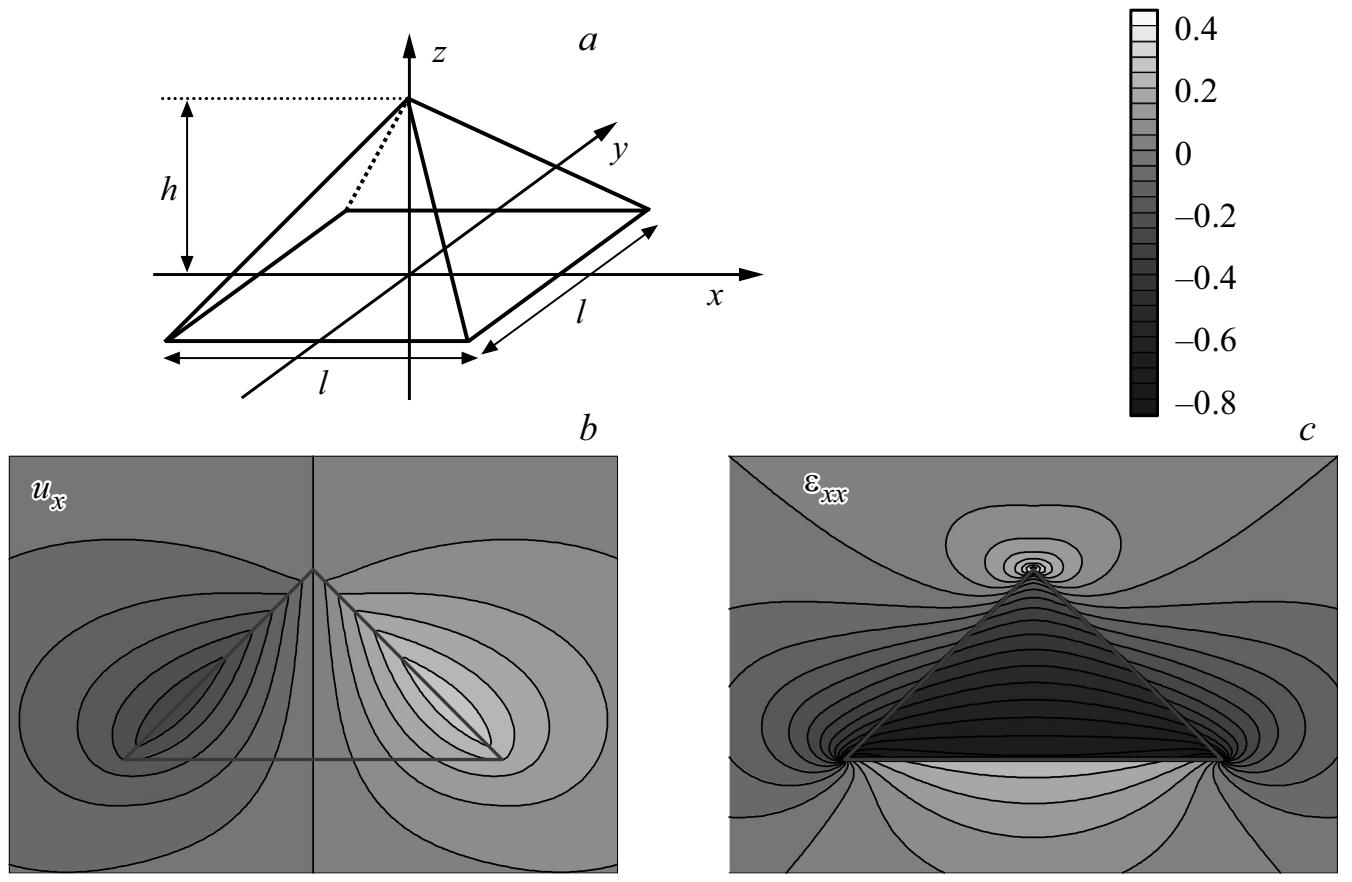

$d$

$e$
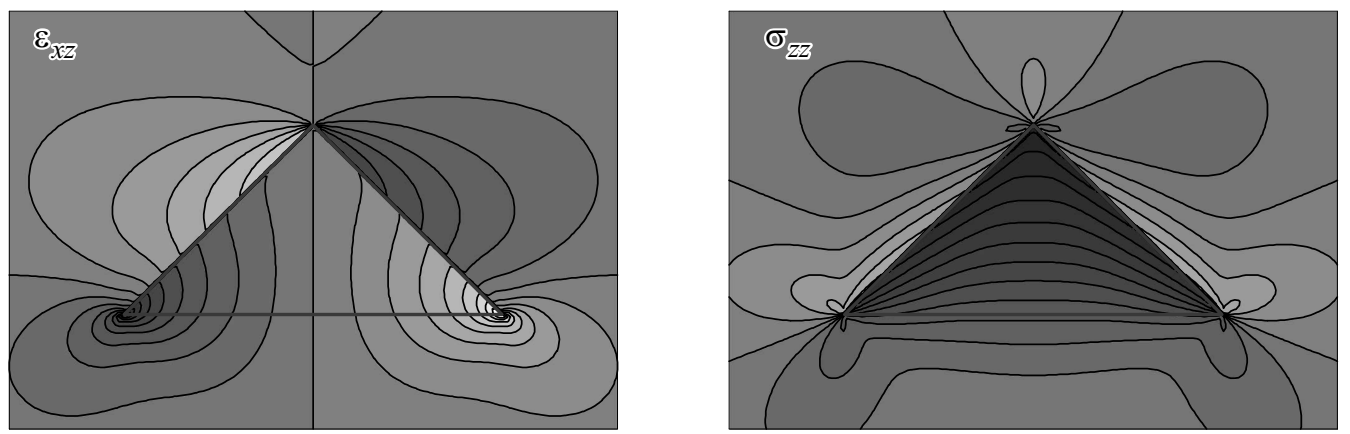

Рис. 3. Компоненты вектора смещения $\mathbf{u}_{x}(\mathbf{r})$, тензора деформации $\varepsilon_{x x}(\mathbf{r}), \varepsilon_{x y}(\mathbf{r})$ и тензора напряжений $\sigma_{z z}(\mathbf{r})$ для включения пирамидальной формы с собственной деформацией, имеющей характер растяжения по оси $X\left(\varepsilon_{x x}^{(0)}>0\right.$, остальные компоненты $\varepsilon_{i j}^{(0)}$ равны нулю). Указанные компоненты приведены в сечении плоскостью $y=0$, темным треугольником показана граница включения. Значения тензора деформации нормированы на $\varepsilon_{x x}^{(0)}$, тензора напряжений - на $\mu \varepsilon_{x x}^{(0)}$, вектора смещения - на $h \varepsilon_{x x}^{(0)}$.

(рис. 3,a). Размер основания $l$ и высота пирамиды $h$ соотносятся как $l=2 h$. Коэффициент Пуассона $v$ принят равным 0.25 . Как и на рис. $1, b, d$, собственная деформация внутри пирамидального включения имела только одну ненулевую компоненту $\varepsilon_{x x}^{(0)}>0$.

Этот расчет можно рассматривать как численный тест корректности полученных в данной работе аналитических результатов. Действительно, мы проверили, что найденные величины $\mathbf{u}(\mathbf{r}), \varepsilon_{i j}(\mathbf{r})$ и $\sigma_{i j}(\mathbf{r})$ обладают следующими свойствами: (i) они стремятся к нулю при $|\mathbf{r}| \rightarrow \infty$; (ii) функция $\mathbf{u}(\mathbf{r})$ непрерывна на границе включения (рис. $3, b$ ); (iii) $\varepsilon_{i j}$ выражается через производные и согласно (2); (iv) $\sigma_{i j}$ и $\varepsilon_{i j}$ связаны посредством закона Гука (4); (v) производные $\sigma_{i j}$ удовлетворяют уравнению равновесия (3); (vi) компоненты тензора деформации $\sigma_{i j}$, ортогональные к какой-либо грани поверхности включения, непрерывны на этой грани например, компоненты $\sigma_{x z}=2 \mu \varepsilon_{x z}$ (рис. $3, d$ ) и $\sigma_{z z}$ (рис. 3,e) на нижней грани. Эти свойства в совокупности гарантируют, что найденные нашим методом функции $\mathbf{u}(\mathbf{r}), \varepsilon_{i j}(\mathbf{r})$ и $\sigma_{i j}(\mathbf{r})$ действительно являются решениями уравнений теории упругости.

\section{4. Заключение}

Полученные результаты можно подытожить следующим образом. Найдены аналитические выражения для вектора смещения $\mathbf{u}(\mathbf{r})$ [формулы (8) и (30)], тензора деформации $\varepsilon_{i j}(\mathbf{r})$ [формулы (9) и (33)] и тензора Эшелби $S_{i j k l}(\mathbf{r})$ [формулы (11) и (33)] в случае, когда включение в упруго-изотропной бесконечной среде имеет форму многогранника. Собственная деформация $\varepsilon_{i j}^{(0)}$ (например, рассогласование кристалических решеток) предполагается постоянной внутри включения, но не обязательно гидростатической. Найденные выражения 
описывают деформацию как внутри включения, так и в его окружении. Константы $p_{i j k l}^{(\beta)}, q_{i j k}^{(\gamma)}, v_{i j k l m n}^{(\beta)}, w_{i j k l m}^{(\gamma)}$, входящие в эти формулы, определяются ориентацией граней и ребер поверхности включения согласно (31), (32), (34) и (35). Сложная трехмерная конфигурация поля упругой деформации (а также вектора смещения) сводится к комбинации простых функций $\Omega_{\alpha}, L_{\beta}$ и $\Lambda_{\beta}$, имеющих наглядную физическую и геометрическую интерпретацию. Следует заметить, что в случае гидростатической собственной деформации $\left(\varepsilon_{i j}^{(0)} \propto \delta_{i j}\right)$ выражения для $\mathbf{u}(\mathbf{r})$ и $\varepsilon_{i j}(\mathbf{r})$ значительно упрощаются и приобретают вид, рассмотренный в статьях [21,5].

Работа профинансирована Российским научным фондом (грант 14-12-00931 П).

\section{Список литературы}

[1] J.D. Eshelby. Proc. Roy. Soc. London A 241, 376 (1957).

[2] T. Mura. Micromechanics of Defects in Solids. Springer (1987).

[3] I.A. Ovid'ko, A.G. Sheinerman. Rev. Adv. Mater. Sci. 9, 17 (2005).

[4] B.N. Kuvshinov. Int. J. Solids Structures 45, 1352 (2008).

[5] A.V. Nenashev, A.V. Dvurechenskii. J. Appl. Phys. 121, 125102 (2017)

[6] C. Pan, Q. Yu. Int. J. Mech. Sci. 126, 142 (2017).

[7] M. Grundmann, O. Stier, D. Bimberg. Phys. Rev. B 52, 11969 (1995).

[8] C. Pryor. Phys. Rev. B 57, 7190 (1998).

[9] O. Stier, M. Grundmann, D. Bimberg. Phys. Rev. B 59, 5688 (1999).

[10] A.V. Dvurechenskii, A.V. Nenashev, A.I. Yakimov. Nanotechnology 13, 75 (2002).

[11] Г.Л. Бир, Г.Е. Пикус. Симметрия и деформационные эффекты в полупроводниках. Наука, М. (1972).

[12] C.G. Van de Walle. Phys. Rev. B 39, 1871 (1989).

[13] A.F. Zinovieva, A.I. Nikiforov, V.A. Timofeev, A.V. Nenashev, A.V. Dvurechenskii, L.V. Kulik. Phys. Rev. B 88, 235308 (2013).

[14] H.J. Chu, E. Pan, J.J. Ramsey, J. Wang, C.X. Xue. Int. J. Solids Structures 48, 673 (2011).

[15] L. Ma, A.M. Korsunsky. Int. J. Solids Structures 51, 4477 (2014).

[16] G.J. Rodin. J. Mech. Phys. Solids 44, 1977 (1996).

[17] H. Nozaki, M. Taya. J. Appl. Mech. 68, 441 (2000).

[18] P. Offermans, P.M. Koenraad, J.H. Wolter, K. Pierz, M. Roy, P.A. Maksym. Phys. Rev. B 72, 165332 (2005).

[19] M. Stoffel, A. Malachias, A. Rastelli, T.H. Metzger, O.G. Schmidt. Appl. Phys. Lett. 94, 253114 (2009).

[20] A. Picco, E. Bonera, F. Pezzoli, E. Grilli, O.G. Schmidt, F. Isa, S. Cecchi, M. Guzzi. Nanoscale Res. Lett. 7, 633 (2012).

[21] A.V. Nenashev, A.V. Dvurechenskii. J. Appl. Phys. 107, 064322 (2010).

[22] И.Е. Тамм. Основы теории электричества. Наука, М. (1976).

[23] A. Van Oosterom, J. Strackee. IEEE Transact. Biomed. Eng. BME-30, 125 (1983).

[24] R.A. Werner, D.J. Scheeres. Celestial Mech. Dynam. Astronomy 65, 313 (1996).

[25] A.V. Nenashev, A.V. Dvurechenskii. arXiv:0707.2183 (2007).

Редактор Т.Н. Василевскал 